\title{
Tiempo es poder: envejecimiento y control del espacio público en un barrio de clase media tradicional en Lima*
}

\section{Omar Pereyra}

Pontificia Universidad Católica del Perú

Sección Sociología

\section{RESUMEN}

Este artículo estudia el efecto del envejecimiento de los vecinos sobre las organizaciones locales en San Felipe, un barrio de clase media en Lima, Perú. Ilustro el efecto de este fenómeno usando el caso del control del espacio público en el barrio. Para esta investigación realicé observación participante durante un año. En ese periodo, observé la dinámica de las asambleas locales, entrevisté a 46 vecinos de distintas caracteristicas y fui testigo de una gran cantidad de situaciones y controversias entre vecinos en los espacios públicos de San Felipe. Encuentro que los adultos mayores son los que imponen su punto de vista respecto del destino del barrio. Dicho resultado es sorprendente, pues los adultos mayores no son ni el grupo demográficamente más importante, ni el de mayores recursos. Sostengo que ello ocurre porque los adultos-mayores transforman el tiempo (un recurso escaso para los adultos jóvenes, pero ampliamente disponible para los adultos-mayores) en poder organizacional.

\footnotetext{
Este artículo tiene su origen en mi tesis doctoral en Sociología en Brown University. Aunque este artículo no es parte ni de la tesis doctoral ni del libro que surgió a partir de aquella (Pereyra, 2015), las ideas expuestas tienen su origen en la discusión y comentarios que recibí de mi comité evaluador. Por ello quisiera agradecer a John Logan, Hilary Silver, José Itzigsohn, David Lindstrom y Mary Fennell. Agradezco también a los evaluadores anónimos de la versión preliminar de este manuscrito por sus comentarios y sugerencias.
} 
Con dicho poder organizacional, los adultos mayores logran influir en los funcionarios municipales, quienes no solo defienden el punto de vista de los adultos mayores respecto del espacio público, sino que además lo transforman en concordancia con dicho criterio.

Palabras clave: clases medias, barrio, organización local, envejecimiento, América Latina.

\title{
Time is Power: Aging and Control of Public Space in a Traditional Middle Class Neighborhood in Lima
}

\author{
ABSTRACT
}

In this article, I study the effect of aging of neighbors on local organizations in San Felipe, a middle-class neighborhood in Lima, Peru. I elaborate on this effect by using the case of the control of public space in the neighborhood. I conducted participant observation during a year. During that year, I observed the dynamics of local organizations 'meetings; I interviewed 46 residents of different characteristics; and I observed a large amount of situations and controversies among actors in San Felipe's public space. I find that senior residents are the ones who impose their point of view about the neighborhood's fortune. This result is surprising considering that senior residents are neither the most numerous group in the neighborhood, neither the one with higher resources. I claim that that happens because senior residents transform time (a scarce resource for young-adult neighbors, though abundant for the senior neighbors) into organizational power. With that organizational power, senior residents are able to influence on the municipality's functionaries who not only defend the discourse of senior residents regarding the use of public space, but also transform it according to this discourse.

Keywords: Middle classes, neighborhood, local organization, aging, Latin America. 


\section{INTRODUCCIÓN}

Las ciudades latinoamericanas se han caracterizado, desde la década de 1940, por un crecimiento explosivo debido a la migración del campo a la ciudad y al fenómeno de la urbanización informal periférica (Portes y Walton, 1976; Roberts, 1978). No debemos dudar de que la urbanización periférica por la pobreza continúa siendo uno de los rasgos estructurales fundamentales de las ciudades latinoamericanas y, por tanto, un tema central de la investigación urbana. Sin embargo, nuevos procesos y fenómenos aparecen en las ciudades, lo que amplía la agenda de los investigadores. Quiero resaltar en este artículo dos grandes procesos de importancia central para las ciudades latinoamericanas contemporáneas. En primer lugar, existe un crecimiento significativo de las clases medias en la mayoría de países latinoamericanos: de un $20 \%$ de la población en la década de 1980 a un $45 \%$ en la actualidad, sobre la base de medidas que combinan criterios de ingreso y ocupación (Franco, Hopenhayn y León, 2010). Paralelamente, investigaciones en los ámbitos regional y nacional sugieren que las clases medias latinoamericanas están volviéndose más heterogéneas en términos de estilo de vida y consumo (Arellano y Burgos, 2010; Franco, Hopenhayn y León, 2010); en términos de clase de origen debido a un proceso de movilidad social ascendente de hijos de migrantes (Arellano y Burgos, 2010; Franco, León y Atria, 2007; Portocarrero, 1998); en términos de color de piel (Arellano y Burgos, 2010; Da Silva y Reis 2011; Portocarrero, 1998), e incluso en términos de repertorios de evaluación o las fronteras simbólicas que se erigen en el momento de clasificar a otras personas (Méndez, 2008; Pereyra, 2015).

Fenómenos asociados a dicho crecimiento de las clases medias en las ciudades son la transformación de antiguos barrios populares que hoy (por movilidad social de sus residentes) se convierten en barrios de clase media, las transformaciones 
urbanas asociadas a este grupo como la aparición de condominios cerrados y la gentrificación, y la aparición de nuevos movimientos urbanos de los no pobres o interclasistas, como es el caso de los movimientos ambientalistas, por el trasporte, por el espacio público, entre otros. En segundo lugar, a pesar de que América Latina continúa en el período llamado el «bono demográfico» (cuando la población en edad de trabajar es mayor que la población dependiente), empieza a notarse un proceso de envejecimiento (Cotlear, 2011). Los estimados sugieren que la población adulta mayor es actualmente el grupo de mayor crecimiento en la región: en los últimos cincuenta años la población más joven (de 0 a 14 años) aumentó en 2,5 veces, la población adulta (de 15 a 59 años) en 3,8 veces, mientras que la población adulta mayor aumentó 5,8 veces. En los próximos cincuenta años, se espera que el primer grupo disminuya en 17\%, el grupo adulto continuaría creciendo en un 33\%, y el grupo adulto mayor se cuadriplicaría (Saad, 2011, p. 52). Para el año 2040, el peso porcentual de la población adulta mayor alcanzaría al de la población más joven, y para 2050 la superaría en 30\%. Este envejecimiento plantea retos nuevos para nuestras sociedades, las cuales tienen que prepararse para una situación en la cual los adultos mayores devendrán paulatinamente en el grupo mayoritario. Este proceso transformaría el tipo de demandas políticas $\mathrm{y}$, por supuesto, la infraestructura y servicios de nuestras ciudades.

Este estudio se centra específicamente en las consecuencias del envejecimiento en el uso del espacio público en un barrio de clase media tradicional. Para este estudio se escogió a la Residencial San Felipe, un barrio icónico de clase media en Lima, Perú. Siguiendo el trabajo de Centner (2010; 2013), tomo el marco teórico desarrollado por Bourdieu (1984) para entender las relaciones entre grupos, extendiéndolo para el estudio del espacio público en San Felipe. De este modo, se entiende al espacio público como un lugar de conflicto entre distintos discursos y prácticas por darle un carácter determinado; es decir, una búsqueda de una «distinción del lugar» (Centner, 2013). En este conflicto colisionan dos grandes visiones sobre cómo debiera ser el espacio público y un barrio de clase media: por un lado, un discurso de la clase media tradicional envejecida que concibe al espacio como ornamental y con un código de conducta más funcional; y por otro un discurso más propio de los jóvenes adultos (tanto de clase media tradicional como de la llamada «nueva clase media») que conciben a este espacio como uno de libre acceso, de diversidad de funciones y abierto a la espontaneidad.

El estudio muestra dos conclusiones sugerentes. En primer lugar, en cuanto al conflicto por el espacio público en el barrio, la división entre «clase media tradicional» $\mathrm{y}$ «nueva clase media» es menos relevante que la frontera generacional. 
En segundo lugar, respecto del poder en el barrio, aparece una paradoja: si bien los residentes mayores no son la mayoría y sus recursos económicos son más limitados, estos se convierten en el grupo más poderoso, llegando a imponer su visión sobre el espacio público.

\section{REVISIÓN DE LA LITERATURA: ESPACIOS PÚBLICOS EN BARRIOS}

Cuando se piensa en el espacio público, generalmente lo asociamos con características como la libertad de acceso, el respeto a la diferencia y la libertad de acción, o al menos a la espontaneidad (Borja, 2003; Harvey, 2006). Debe notarse también que, cuando se habla de espacio público, generalmente pensamos en las grandes plazas, veredas o avenidas de la ciudad. Sin embargo, aparece un problema cuando pensamos en lugares que en principio son definidos como espacios públicos, pero que tienen una dinámica más local, como es el caso de las calles o parques en los barrios; todos ellos con un control más fuerte de los vecinos. Precisamente, para entender estos espacios, necesitamos marcos teóricos distintos de los generados por las grandes aproximaciones al espacio público.

Una candidata es la escuela de la Economía Política Urbana (Logan y Molotch, 1987), la cual tiene un cuerpo teórico desarrollado para estudiar el conflicto urbano tanto en el barrio como en la ciudad en general. Desde esta perspectiva se entiende al «lugar» o barrio como un recurso estratégico asociado a oportunidades de vida para sus residentes. En este sentido, los actores urbanos compiten por el acceso a mejores lugares en un sistema de «estratificación de lugares», así como por mantenerlos o transformarlos. Sin embargo, se puede decir que esta es una perspectiva fundamentalmente materialista.

Examinar la idea del conflicto urbano por el lugar desde el marco desarrollado por Bourdieu, amplía las posibilidades en algunas direcciones. Desde esta perspectiva podemos pensar que el lugar (barrio o comunidad) tiene significados adscritos o construidos que escapan a las lógicas de «valor de uso» o «valor de cambio». Precisamente, algunos de los conflictos por la defensa del lugar tienen más que ver con la defensa del carácter o el simbolismo del lugar. Es decir, bastante del conflicto por el lugar tiene que ver con la «distinción» del lugar y, con ello, con la imagen y estilo de vida de sus residentes (Centner, 2010).

Pero además, siguiendo a Bourdieu, Centner (2013) sugiere que la idea de «derecho a la ciudad», generalmente asociada al conflicto entre capital y ciudadanos (Borja, 2003; Harvey, 2006; Lefebvre, 1996 [1967]), debiera ser puesta en cuestión. A partir del estudio de transformaciones urbanas en Buenos 
Aires, Brasil y Turquía, el autor menciona que es difícil, o incluso ingenuo, pensar en una idea única o abstracta de «derecho a la ciudad». Lo que muestran los casos es que en estos proyectos de transformación urbana existen conflictos entre visiones de «derecho a la ciudad» encarnadas en discursos sobre cómo debiera ser la ciudad. Al igual que otras nociones, la noción de «derecho a la ciudad» no es una categoría absoluta, sino que es una categoría en disputa y transformación (Centner, 2013). El objeto de estudio que se abre es ahora el conflicto entre diversos actores (incluyendo a los estudiosos de la ciudad y los activistas «progresistas») por definiciones y materializaciones del espacio público.

El caso del espacio público en la Residencial San Felipe es interesante por estar atravesado por el proceso de heterogeneización de la clase media, encarnada tanto en la típica división de estatus entre las llamadas «clase media tradicional» y la «nueva clase media» como en la nueva brecha generacional. San Felipe nos permite evaluar la relevancia de estas divisiones internas, así como mecanismos inesperados para hacer prevalecer un punto de vista en un barrio. Explorar estas fracturas y mecanismos nos permitiría entender mejor el poder en barrios de clase media.

\section{CASO DE ESTUDIO: SAN FELIPE, UN BARRIO DE CLASE MEDIA}

San Felipe fue creado en 1967 como un proyecto de vivienda dirigido específicamente a la clase media limeña; entendiendo por esta a profesionales independientes, trabajadores de cuello blanco del sector público y privado, así como oficiales de las fuerzas armadas y de la policía nacional. Dos características de San Felipe hacen de este barrio un espacio privilegiado para este estudio. En primer lugar se encuentra su estructura arquitectónica y organizativa. Siguiendo los principios de la arquitectura funcionalista, San Felipe cuenta con 33 edificios de departamentos unifamiliares y una gran cantidad de espacios comunes, como parques, estacionamientos y plazas. Este diseño hace que San Felipe desarrolle una vida de barrio intensa por las relaciones cotidianas que crea entre sus vecinos. Pero además, esta estructura arquitectónica lleva a una vida organizacional sólida y fácil de observar: mientras el problema del mantenimiento cotidiano de los edificios ha llevado a que cada uno cuente con una Junta de Propietarios, para el caso de los espacios comunes y del barrio en general existen tres Juntas Vecinales que congregan a los presidentes de las Juntas de Propietarios de los edificios. 
En segundo lugar, dado que San Felipe tiene ya más de cuarenta años de antigüedad, varios de sus vecinos fundadores son actualmente adultos mayores ${ }^{1}$. Hoy en día la mayoría de los vecinos de San Felipe son los hijos de estos vecinos pioneros (hoy adultos entre los 35 y 50 años) y las nuevas familias jóvenes que han venido a ocupar los departamentos dejados paulatinamente por la primera generación de sanfelipanos. Esta característica particular hace que, de acuerdo con los datos del último censo nacional ${ }^{2}$, el porcentaje de adultos-mayores en San Felipe (23,1\% mayores de 65 años) sea alto en comparación al de otros barrios aledaños $\left(15,2 \%\right.$ en el distrito) y de la ciudad en general $(6,73 \%)^{3}$. De este modo, San Felipe dista de ser un barrio «promedio» en términos de su composición demográfica. Es más bien un barrio en donde el envejecimiento es más pronunciado que en el resto de la ciudad. Precisamente este rasgo exagerado de San Felipe permite explorar de forma más clara el efecto del envejecimiento en las organizaciones locales y en la vida cotidiana del barrio.

La ecología de San Felipe ha experimentado los mismos cambios que la clase media de Lima en general. Muchas de sus familias más nuevas son jóvenes, y vienen de una trayectoria de movilidad social ascendente. San Felipe es un espacio que permite ver precisamente el encuentro entre la llamada «clase media tradicional» (los vecinos adultos mayores y sus hijos, hoy adultos jóvenes con sus propias familias) y la «nueva clase media» (los nuevos vecinos adultos jóvenes con una trayectoria de movilidad social ascendente). Ello lleva, por ejemplo, a que muchos de los vecinos mayores de San Felipe consideren al ingreso de nuevos vecinos

1 Aunque oficialmente se denomina adulto mayor a las personas mayores de sesenta años, en este texto defino a estas personas como mayores de 65 por ser la marca oficial de ingreso a la jubilación. El ingreso a la jubilación es una marca más importante que la edad cronológica en el sentido que significa comúnmente no solo una reducción significativa en los ingresos, sino también un cambio radical en la rutina cotidiana al salir del mundo del trabajo y por generar, en consecuencia, una gran cantidad de tiempo no ocupado.

2 Instituto Nacional de Estadística e Informática - INEI. Censos Nacionales de Población y Vivienda, 2007. Base de datos en el nivel de manzana.

3 Debería esperarse que el porcentaje de población adulta mayor sea más alto actualmente. Sin embargo, es difícil hacer un estimado de la composición demográfica actual. Por un lado, si bien la población envejece y ello aumenta el porcentaje de población por encima de los 65 años, algunos adultos mayores fallecen, y sus departamentos son ocupados por vecinos más jóvenes, quienes los heredan o los compran a los herederos. También se ha encontrado durante el trabajo de campo que algunos adultos mayores llegan a vivir a los departamentos de San Felipe luego de vender sus casas antiguas, buscando un lugar más seguro y con varios servicios «a la mano» (tiendas, peluquerías, restaurantes, parques, etc.). También se ha encontrado que algunos adultos mayores son llevados a San Felipe por sus hijos a vivir con ellos para poder cuidarlos. 
como señal de cierto «deterioro» del barrio. Sin embargo, los vecinos mayores, dada su situación de jubilados, hoy en día tienen ingresos menores a los de los vecinos más jóvenes. Empero, el rasgo que hace de San Felipe un barrio de clase media contemporánea es que, si bien sus familias trabajan en ocupaciones más diversas que en sus inicios (incluyendo ahora a los jubilados y pequeños empresarios), estas tienen actualmente, en su gran mayoría, credenciales profesionales técnicas o universitarias. Es decir, San Felipe es barrio de un sector muy particular de los que constituyen las clases medias: el de los profesionales de rango medio. No son ni profesionales altamente calificados o que ocupan posiciones altas en grandes empresas, ni pequeños comerciantes o empresarios no profesionales que se encuentran un poco por encima de la pobreza.

\section{METODOLOGÍA}

Este artículo estudia el conflicto por el espacio público en San Felipe. Como puede desprenderse de la descripción de San Felipe, este no es un barrio «típico». Por el contrario, es un barrio excepcional en muchos sentidos. En primer lugar, su estructura arquitectónica y su vida social hacen de San Felipe un espacio con una vida social más intensa que la de cualquier otro barrio de clase media, generalmente con residentes menos anclados en la localidad y con relaciones más impersonales entre sus vecinos. Del mismo modo, dado su origen como proyecto de vivienda, el envejecimiento en San Felipe es más acentuado que el de otros barrios. Desde este punto de vista, San Felipe no es exactamente un caso «atípico», sino un caso con rasgos exagerados que son de especial interés, incluso estratégicos, para mi pregunta de investigación ${ }^{4}$.

Para entender el espacio público en San Felipe realicé observación participante durante un año. Durante ese año, viví en San Felipe y me convertí en un vecino interesado en problemas de participación local. Descubrí rápidamente que los adultos mayores eran quienes tenían control sobre el espacio público; no solo vigilando la conducta de las personas, sino además promoviendo transformaciones en su infraestructura de acuerdo con su visión de cómo debía ser el barrio. Dicho hecho es sorprendente, pues los adultos mayores no son ni el

4 Considero, siguiendo a Burawoy (1998), que no tiene sentido hablar de «casos típicos» en los estudios cualitativos. Lo lógica que guía la investigación cualitativa es la de entender el caso en sí mismo y su conexión con las fuerzas o procesos mayores que lo afectan (Comaroff, 1982). En consecuencia, la lógica de este estudio no es buscar representatividad o generalizabilidad, sino entender cómo se desarrollan dinámicas, mecanismos o procesos. 
grupo demográficamente más importante, ni el grupo de mayores recursos (dos fuentes de poder consideradas como centrales en la literatura sobre barrios y comunidades). La respuesta a esta paradoja se encuentra en que los adultosmayores tienen el control de las organizaciones locales, y este control sobre las organizaciones les da influencia sobre los funcionarios municipales. Mi objetivo de investigación fue entonces explorar esta conexión entre organizaciones locales y el control del espacio público. Para ello exploré dos mecanismos principales: primero, ver cómo los adultos mayores tomaban el control de las organizaciones locales, y segundo, ver cómo usaban este poder organizacional para el control y transformación del espacio público.

Para entender el proceso por el cual los adultos mayores toman el control de las organizaciones locales, realicé dos actividades paralelas. En primer lugar, observé todas las asambleas de Juntas de Propietarios a las que tuve permiso de asistir a lo largo del trabajo de campo (un total de veintisiete asambleas). En estas reuniones tomé nota de quiénes eran los vecinos que asistían, los temas de conversación previos a las reuniones, los temas que se discutían en las reuniones $\mathrm{y}$, sobre todo, del comportamiento de los participantes y el tipo de interacciones que se desarrollaban.

Para entender el uso del poder organizacional para el control del espacio público, observé directamente las reuniones de la organización mayor del barrio, las Juntas Vecinales, y su relación con los funcionarios de la Municipalidad. En ellas registré el tipo de temas y demandas que se discutían respecto del espacio público en San Felipe. Finalmente, registré por observación directa una gran cantidad de situaciones y controversias en torno al uso del espacio público (como niños jugando fútbol en el parque, montando skate en las veredas, trepando árboles, y gente comiendo en los parques), así como las discusiones originadas por algunas transformaciones físicas del espacio (como la instalación de cercos y el plantado de arreglos florales en los parques). Complementé este ejercicio de observación con conversaciones informales con los actores involucrados en estas situaciones.

\footnotetext{
De los 33 edificios de San Felipe, tuve permiso de asistir a las reuniones de 21 Juntas de Propietarios. De estas 21 Juntas de Propietarios, doce no tuvieron reuniones durante el año del trabajo de campo. En ellas los miembros de la Presidencia (generalmente el presidente solo, y a veces una terna formada por el presidente, el tesorero y el secretario) actuaban con libertad, pero rindiendo cuentas de los gastos a los vecinos por medio de comunicados. De las nueve Juntas de Propietarios restantes, tres tuvieron solo una reunión durante el año, y las otras seis tuvieron más de dos reuniones.
} 
Finalmente, para hacer la conexión entre estos dos grandes mecanismos, realicé 46 entrevistas semiestructuradas a distintos tipos de vecinos (adultos jóvenes, adultos y adultos mayores; hombres y mujeres; nuevos y antiguos). Con respecto a la participación local, las entrevistas giraron en torno a tres temas principales: respecto de sus motivos para participar o no en las reuniones de las Juntas de Propietarios de los edificios, respecto de la forma cómo ven o evalúan a dichas asambleas, y respecto de sus experiencias personales en ellas. Con relación al control del espacio público en el barrio, pregunté por sus visiones (o «enmarcados» $\left.{ }^{6}\right)$ del espacio público, así como respecto de su posición en situaciones o controversias sobre su uso.

\section{CLASES MEDIAS Y PODER EN CONTEXTOS BARRIALES}

Una característica principal del espacio público de San Felipe es que se encuentra regido por un discurso sobre el comportamiento correcto en público que señala límites sobre lo que se puede hacer en él y lo que no. Dicho discurso es el de los vecinos adultos mayores, que se sienten no solo autorizados para controlar directamente el espacio (decir qué es correcto y qué no lo es), sino que además controlan las organizaciones locales e influyen sobre los agentes municipales para hacerlo respetar.

\section{Dos visiones sobre el espacio público en San Felipe}

En comparación con otros barrios, San Felipe tiene una gran cantidad de áreas verdes. Por ello, para los sanfelipanos, los parques y jardines son elementos importantes de distinción del barrio. En consecuencia, el mantenimiento de estas áreas verdes es un tema importante de conversación y preocupación entre los vecinos. Entre los sanfelipanos, existen dos discursos principales respecto de las áreas verdes y el espacio público en general. Uno es el de los adultos mayores. Para ellos, el espacio público se debe organizar en torno a tres principios generales. El primero es que el espacio es moral, refiriéndose a que debe mantenerse

6 De modo general un «marco» se refiere a «... un esquema interpretativo que simplifica y condensa el mundo exterior" a través de un proceso de distinción y codificación de objetos, situaciones, eventos, experiencias y secuencias de actos respecto al presente o pasado de uno» (Snow y Benford, 1992, p. 137. Traducción mía). Los «marcos» nos permiten no solo clasificar determinados fenómenos, sino que nos llevan o motivan a optar por secuencias de acción diferenciadas (Small, 2004). 
un código de conducta en él. Vestirse adecuadamente y comportarse de forma amable y respetuosa es importante no solo para mostrar una imagen adecuada de uno mismo, sino para preservar el orden y la moral pública. Del mismo modo, el pudor es importante, por lo que los besos prolongados y tocamientos entre parejas también son mal vistos. El segundo principio es que el espacio es funcional, es decir que hay áreas que están diseñadas para algunas cosas pero no para otras. Por ejemplo, los estacionamientos son para los autos, las veredas son para caminar o tener pequeñas conversaciones, las bancas son para sentarse y, en el caso de los parques, el espacio es ornamental. Sobre la base de estos principios, «actividades fuera de lugar» (como comer o beber en público, montar skate, caminar en el césped, o sentarse en un rincón a conversar en grupo) son disruptivas del orden público y deben ser evitadas.

El segundo discurso sobre el espacio público en San Felipe es el de los vecinos adultos jóvenes, tanto antiguos como nuevos. Aunque los adultos jóvenes también valoran sus áreas verdes, consideran que el cuidado de aquellas se ha tornado obsesivo en los últimos años. Los adultos jóvenes mencionan que los espacios públicos de San Felipe deberían ser más «como espacios públicos», refiriéndose con ello a que los niños deberían poder jugar en los parques y los jóvenes deberían poder reunirse a conversar y pasar el rato en las veredas o gradas. No consideran que los chicos que montan skate o que juegan fútbol en el parque sean una molestia o señal de deterioro del barrio, sino que, por lo contrario, ello es lo que da «vida» a San Felipe.

Como mencioné, es el discurso de los adultos mayores el que acaba imponiéndose. ¿Cómo es posible este resultado? Existen dos formas principales por las cuales los adultos mayores logran imponer su visión del espacio público: el control directo por medio de interacciones personales (sea un adulto mayor o un funcionario municipal llamando la atención a un infractor), y el control indirecto por medio de la instalación de artificios que restringen el uso del espacio (principalmente de las áreas verdes). Respecto del control directo, es importante considerar que los adultos mayores, por su situación de retiro, son el grupo cuya presencia es más visible y permanente en San Felipe. Esto convierte a los adultos mayores en guardianes de facto del espacio. Cuando los adultos mayores ven a grupos de skaters saltando en las veredas o gradas, o cuando ven a grupos de chicos jugando fútbol en alguno de los parques, se les acercan y les dicen que eso no está permitido en San Felipe. En estos casos, los más chicos generalmente dejan de jugar y van a otro lado en donde siguen jugando hasta que aparezca otro adulto mayor y los haga salir. Sin embargo, también es posible que los chicos 
no hagan caso y reclamen su derecho al uso del espacio. En esta situación, los adultos mayores necesitan refuerzos. Estos llegan desde los funcionarios de la Municipalidad, particularmente por medio de la intervención de serenos (guardias municipales). Sin embargo, para que los funcionarios de la Municipalidad hagan suyo el punto de vista de los adultos mayores, estos últimos deben convertirse en los vecinos más influyentes, y como vimos, esto ocurre a pesar de no ser el grupo mayoritario en San Felipe.

\section{El control de las organizaciones locales en San Felipe}

El tamaño de los grupos y sus recursos económicos son, por lo general, buenos indicadores la distribución del poder en los barrios. Sin embargo, en San Felipe estas características no importan mucho. De hecho, ocurre lo contrario: el grupo más pequeño y con menores recursos, el de los adultos mayores, es el grupo más poderoso, y este controla y transforma el barrio según sus intereses. Este resultado ocurre por dos motivos principales. Primero, los adultos mayores tienen tiempo (un recurso escaso para los vecinos más jóvenes) y gracias a ello, logran el control de las organizaciones locales. Segundo, los adultos mayores usan las organizaciones locales para influir en las autoridades locales. Nos interesa en este punto explicar cómo es que los adultos mayores adquieren el control de las organizaciones locales.

La observación continua de las asambleas de las Juntas de Propietarios y las entrevistas a los vecinos de San Felipe revelan dos mecanismos principales que de forma combinada afectan la participación local. Primero, un mecanismo extralocal: la relación de los vecinos con el mercado de trabajo, el cual en el caso de los vecinos más jóvenes (antiguos y nuevos) limita su posesión de tiempo para la participación local. Segundo, un mecanismo intralocal, el comportamiento de los vecinos mayores en las asambleas, el cual desincentiva aún más la participación de los vecinos más jóvenes. De este modo, los vecinos más jóvenes son «sacados» del barrio por sus responsabilidades laborales, pero también practican lo que Hirschman (1970) llama «salida», o en este caso, «renuncian» a la participación en las organizaciones locales.

Este efecto de la disposición diferenciada de tiempo por generaciones resulta interesante para entender el poder en el barrio. De hecho, la literatura sobre organizaciones locales en barrios diversos ha enfatizado más bien que las grandes brechas de desigualdad societal (brechas de ingresos o diferencias de color) se reproducen a nivel local generando la monopolización de las organizaciones 
por cierto grupo y la exclusión de los otros (Graves, 2010; Joseph, 2008; pero ver Elias y Scotson, 1994). Sin embargo, San Felipe muestra más bien un caso en donde, a pesar de la diferencial de ingresos (entre adultos mayores y vecinos más jóvenes), y a pesar de la estigmatización que recae sobre los vecinos nuevos (debido a su origen social), la invitación a participar en las organizaciones es abierta y sin restricciones. De hecho, los adultos mayores se quejan continuamente de la baja participación de los más jóvenes en las reuniones. Lo que ocurre es que ni la brecha de origen social (pues ni los vecinos adultos jóvenes antiguos ni los nuevos participan por igual), ni la brecha de ingresos (pues los vecinos de menores ingresos, los adultos mayores, son los que acaban controlando las organizaciones) son relevantes.

Al preguntar a los vecinos adultos jóvenes sobre su poca participación en las asambleas, sus respuestas están comúnmente asociadas con sus rutinas de trabajo, que no les dejan tiempo para participar. Los adultos jóvenes describen su rutina típica como bastante cargada de actividades: salen de la casa a las 7:30 a.m., dejan a los niños en el colegio y luego van al trabajo, están en el trabajo la mayor parte del día, y finalmente regresan a casa entre las ocho y las nueve de la noche. Es también común que estos tengan un segundo trabajo (por ejemplo, consultorías o negocios propios) que hacen que sus jornadas de trabajo se extiendan. Vecinos jóvenes, tanto antiguos como nuevos, mencionan que, al final de su rutina de trabajo, solo quieren quedarse en casa y pasar el tiempo con la familia. Se agrega a ello que, a diferencia de la generación de los adultos mayores, en las familias más jóvenes tanto hombres como mujeres trabajan, con lo cual el contacto de la familia nuclear con el barrio es menor. La situación es completamente distinta para los adultos mayores que, al ser en su mayoría retirados, tienen más tiempo para participar, y su presencia en las asambleas acaba siendo mayoritaria.

Aun así, algunos adultos jóvenes entrevistados mencionan que han intentado participar en las asambleas. Sin embargo, es aquí cuando un mecanismo intralocal - el comportamiento de los vecinos mayores en las asambleas y el estilo con que ellas se conducen - termina por desincentivar la participación de los más jóvenes. Un primer punto respecto de cómo se conducen estas reuniones es que, dado que la mayoría de los participantes son adultos mayores, estas son usadas tanto para discutir problemas del edificio como para socializar. Las reuniones generalmente empiezan varios minutos tarde, debido a que los adultos mayores aprovechan estas reuniones para conversar y ponerse al día respecto de las vidas de sus vecinos (acerca de sus hijos o los nietos o acerca de problemas de salud y enfermedades que los acosan). Para los adultos mayores, esta forma de proceder 
no es solo parte de la camaradería que corresponde a una reunión de vecinos sino que, además, estas conversaciones les proporcionan información que les resulta valiosa. Por ejemplo, en estas conversaciones comparten información sobre el funcionamiento del sistema de salud, sobre lugares cercanos donde acudir para medirse la presión o glucosa, o sobre campañas o actividades que realiza la municipalidad. Desde el punto de vista de los vecinos más jóvenes, estas conversaciones son «poco relevantes» y retrasan las discusiones sobre los temas que ellos consideran «importantes».

Otra característica de las asambleas es que las discusiones sobre cuestiones operativas respecto del mantenimiento del edificio muchas veces se salen del tema. Esto ocurre principalmente por dos motivos. El primero es que los adultos mayores se comportan de una manera bastante formal y ceremonial en estas reuniones. Los adultos mayores, al iniciar las reuniones, suelen repasar los acuerdos de las reuniones anteriores, agregar más puntos a la agenda propuesta inicialmente o proceden varias veces a hacer votaciones. $\mathrm{Al}$ respecto, los vecinos más jóvenes señalan que preferirían una forma más expeditiva y eficiente de conducir las asambleas. Del mismo modo, señalan que los comentarios de los participantes deberían ir directamente al punto en discusión y que debería evitarse hacer referencias a temas que llevan la discusión a otro lado. Segundo, los vecinos más jóvenes también se ven desincentivados de participar por los conflictos entre vecinos que a veces explotan en estas reuniones. Al respecto, los vecinos más jóvenes mencionan que las asambleas «están llenas de las mismas discusiones que duran años de años», las cuales son vistas nuevamente como sin sentido.

De este modo, los vecinos más jóvenes, al no asistir a las reuniones, evitan lo que desde su punto de vista es «perder tiempo». Pero el precio que pagan es doble. Por un lado, al no asistir a las asambleas pierden oportunidades de socializar y, con ello, de formar redes sociales con otros vecinos. Precisamente, los vecinos más jóvenes son bastante atomizados o no forman muchos contactos con otros vecinos; forman en cambio redes pequeñas y dispersas con algunos de sus pares. Por otro lado, acaban aceptando las decisiones que los adultos mayores toman en las asambleas. Por el contrario, al ser la mayoría de los asistentes a las reuniones y al tener más tiempo para la participación, los adultos mayores no solo fortalecen sus relaciones entre ellos, formando redes sociales extensas ancladas en su edificio y en el barrio, sino que también acaban siendo los presidentes de las asambleas ${ }^{7}$. Se puede decir que es

Durante mi año de trabajo de campo en San Felipe, solo encontré dos Juntas de Propietarios en donde los presidentes eran vecinos jóvenes. En ambos casos, estos definieron su posición como 
un resultado relativamente cómodo para los vecinos más jóvenes, dado que la mayor parte del trabajo (y el tiempo) necesario para mantener la infraestructura del edificio funcionando es asumida por los adultos mayores. Sin embargo, el resultado no esperado es que los vecinos más jóvenes no tienen representación en las organizaciones locales, y con ello, su capacidad para influir en las autoridades locales es reducida.

\section{Del control de organizaciones al poder local}

El control de las Juntas de Propietarios permite a los adultos mayores tomar decisiones respecto de los edificios, pero no necesariamente sobre el barrio. Pero es por medio del control de las Juntas de Propietarios que acceden al control de una organización mayor: las Juntas Vecinales, las cuales sí tienen incidencia local. A través de las Juntas Vecinales, los participantes entran en contacto directo con los funcionarios de la municipalidad distrital. Mediante la participación en las Juntas Vecinales, reciben información respecto de las actividades de la municipalidad, mientras que los funcionarios ediles toman nota de los pedidos y reclamos de los vecinos. Por tanto, controlar las organizaciones locales acaba convirtiéndose en un recurso clave para tener contacto e influir en las decisiones de la municipalidad sobre el barrio.

Al igual que en el caso de las Juntas de Propietarios, los adultos jóvenes no aparecen en estas reuniones de las Juntas Vecinales. En este caso, su poca participación se explica porque los miembros de las Juntas Vecinales son en principio los presidentes de las Juntas de Propietarios. Sin embargo, la convocatoria a las reuniones es abierta a todos los vecinos. Pero nuevamente los vecinos más jóvenes no tienen tiempo para ellas, o simplemente no se enteran de los días y horas en los que estas ocurren. De este modo, los reclamos de los adultos mayores son los recogidos por la municipalidad, y con ello, estos acaban siendo considerados como los de los vecinos en general. Los puntos de vista de los adultos jóvenes no aparecen y no son considerados en el momento de toma de decisiones respecto de las alteraciones en el espacio público.

«temporal» y mencionaron que aceptaron su posición como presidentes porque sus edificios se encontraban «en crisis» (en un caso por avería del elevador durante más de tres meses, en otro por una plaga de cucarachas en el edificio). Dado que los vecinos mayores no lograban ponerse de acuerdo en una solución, decidieron entonces tomar el liderazgo. Mencionaron que aceptaron la presidencia con la condición de que su gestión durara solo el tiempo necesario para resolver el problema. En efecto, fueron presidentes por unos cuantos meses, luego de lo cual, se convocó nuevamente a elecciones y se escogió a otro presidente (nuevamente un vecino adulto mayor). 
El control del espacio público no es un problema aislado sino un tema de continua preocupación y discusión en las reuniones de las Juntas Vecinales, así como en conversaciones formales e informales entre vecinos de San Felipe y funcionarios de la Municipalidad. En las reuniones de las Juntas Vecinales, los vecinos discuten continuamente sobre el «problema» de los niños que juegan en el parque y los skaters en las veredas. En reuniones con los funcionarios de la Municipalidad, muestran su descontento con el trabajo de cuidado de los espacios públicos por los serenos, reclaman que se debe contratar a más serenos y que estos deben mostrarse más firmes y con mayor autoridad frente a los chicos. También sugieren y demandan que se instalen objetos que desincentiven el uso del parque, como cercos (para restringir el acceso al parque), arreglos florales en el césped (para evitar que los jóvenes pisen el césped o jueguen en él), arbustos alrededor de los árboles (para evitar que se suban a los árboles), e incluso imágenes religiosas (para evitar que los jóvenes beban en las calles o jueguen a la pelota). Además, se pide la instalación de rampas para sillas de ruedas en lugares en donde existen gradas, así como de bancas para poder descansar.

Los serenos son medianamente efectivos para controlar el espacio público, aunque su intervención suele generar resistencia. Es común en los parques de San Felipe ver a grupos de padres jóvenes jugando con sus hijos pequeños o supervisando a sus hijos si estos son un poco más grandes. En estos casos, su presencia en los parques no es vista como disruptiva por los vecinos mayores ni por los serenos. Pero cuando los niños son más grandes (alrededor de diez años o más), estos suelen salir con sus grupos de amigos, ya sin sus padres. Este es precisamente el grupo que es considerado como potencialmente más «peligroso» para el orden en el espacio público. Sin embargo, estos grupos son generalmente neutralizados por los serenos que piden que «se respete a los vecinos».

La mayoría de las veces, los niños y jóvenes aceptan estas restricciones y acaban sentados en las bancas o gradas. No obstante, hay veces en que los chicos siguen jugando, insistiendo en que ellos también son vecinos y que tienen derecho a usar el espacio ${ }^{8}$. Ante ello, los serenos retroceden en su intento de dispersarlos, pero piden que jueguen de modo más tranquilo para evitar problemas con los vecinos (mayores). Para ello, se quedan en el lugar supervisándolos directamente.

8 En conversación con algunos adultos jóvenes, estos me mencionaron que han instruido a sus hijos para que en una situación como esta le respondan al sereno diciéndole que ellos también son vecinos y que tienen derecho al uso del espacio. También los han instruido para que tomen el nombre del sereno, con el fin de que sus padres presenten la queja ante el capitán de serenazgo, pues parte de su trabajo es cuidar de ellos. 
En estas situaciones, los chicos juegan más despacio y gritan menos, con lo que el juego pierde mucho de su espontaneidad y hasta se vuelve aburrido. Al cabo de un rato, los chicos acaban por ir a buscar otro sitio a donde jugar sin supervisión. Esta forma de controlar el espacio público funciona la mayoría de las veces.

Pero hay situaciones más complicadas, en donde la supervisión de serenos no es eficiente. Precisamente, durante mi trabajo de campo, un grupo de niños empezaron a usar regularmente un área del parque para jugar fútbol en las tardes. Dos veces presencié que los adultos mayores llamaron a los serenos para pedirles que saquen a los niños del parque. En ambas ocasiones los niños se negaron, diciendo que ellos también eran vecinos y que tenían derecho de usar el parque. El caso fue tema de conversaciones informales entre adultos mayores y el presidente de una de las Juntas Vecinales. El tema también apareció (señalado como "problema») en reuniones con funcionarios municipales que visitaban San Felipe. En dichas conversaciones se pedía a los funcionarios municipales que contratasen a más serenos e instruirlos para que sean «más marciales» con los niños. Las conversaciones quedaron en la promesa de ver de dar una solución al problema. En menos de mes, la municipalidad instaló un nuevo arreglo floral en la zona usada para jugar fútbol. También se instaló un perímetro de cactus alrededor de un árbol que a veces era trepado por algunos niños. Además, se asignó de manera permanente la presencia de un sereno en el área, en este caso, para cuidar que los arreglos florales no fuesen vandalizados. En este caso, la instalación de estos objetos no tuvo resistencia ni de los niños y jóvenes ni de sus padres. El grupo de niños no se volvió a reunir en el parque.

Como puede verse, el uso del espacio público es siempre un tema de tensión, y no necesariamente controlado. Sin embargo, el punto de vista de los adultos mayores respecto del mismo es el que acaba prevaleciendo gracias al control y vigilancia de los vecinos y de los funcionarios de la municipalidad.

\section{CONCLUSIONES}

Es necesario resaltar la necesidad de cuestionar y adaptar categorías que no parecen funcionar muy bien cuando hablamos de espacios locales; en específico, «derecho a la ciudad» y «espacio público». En definitiva, estas deben pensarse más allá de la tradicional dicotomía capital-ciudadanos (a partir de la cual gira buena parte de la discusión al respecto). Particularmente estos espacios públicos locales deben pensarse en términos de espacios de conflicto no solo entre capital y residentes, sino también entre residentes y actores externos, e incluso entre los 
mismos residentes. Pero además, deben pensarse más allá de intereses puramente materiales, sino en términos de discursos, narrativas o de imponer una imagen, un estilo de vida o un estatus asociado al lugar. Desde ese punto de vista, el marco teórico desarrollado por Bourdieu — y a partir de este por Centner- aparece como una buena opción para pensar el espacio público como un espacio de conflicto de un tipo más multidimensional.

San Felipe es un barrio bastante particular, y se podría decir que hasta exagerado en cuanto a su proceso de envejecimiento, su densidad organizativa y en la vitalidad de su vida de barrio. Sin embargo, el caso de San Felipe muestra una dinámica que se puede esperar en formas y con intensidades distintas en otros barrios. Pero lo que quiero señalar aquí es precisamente cómo una dinámica mayor (el envejecimiento) se experimenta en un caso particular. A partir de ello, propongo haber encontrado fenómenos y mecanismos que (con variaciones) se pueden esperar en otros casos.

San Felipe es un caso sugerente respecto del impacto del envejecimiento poblacional sobre las organizaciones locales, y por extensión, en el control del espacio público en barrios. El caso nos enseña dos fenómenos principales. Primero, que aunque la división entre «clase media tradicional» y «nueva clase media» atraviesa mucha de la vida cotidiana del barrio, esta tiene una relevancia secundaria en cuanto a la vida organizativa y el control del espacio público. En estos aspectos, la división principal (y con efectos redundantes) es la frontera generacional, la cual se traduce en discursos e intereses distintos respecto del espacio público, en contactos y relaciones diferenciados con el barrio, así como en diferenciales de poder local. Segundo, que la tenencia de tiempo aparece como un recurso central para entender el poder en San Felipe, al menos en lo que respecta al control de las organizaciones locales, y en consecuencia, para tener capacidad de influir en los funcionarios del gobierno local. Dicho control de organizaciones permite no solo alterar el espacio según los intereses y gustos de un grupo, sino además contar con aliados o soporte institucional mayor (en este caso, los funcionarios de la municipalidad), para controlar el espacio cuando el grupo es incapaz de hacerlo por sus propios medios. De este modo, los débiles en términos económicos, demográficos y hasta físicos acaban siendo los más fuertes en el barrio. Inesperadamente, las fuentes tradicionales de poder (recursos económicos, educativos, o el peso demográfico) aparecen como poco significativas con respecto al poder local. Es más bien el tiempo (un recurso del cual los más fuertes suelen carecer, mientras que es abundante entre los más débiles) el que acaba siendo un recurso central en este aspecto. Es decir, en barrios como este, quienes tienen tiempo son quienes potencialmente tienen poder. 
Finalmente, vale la pena extender un poco los hallazgos de este estudio para pensar la ciudad, dado que esta atravesará en las próximas décadas un proceso de envejecimiento acelerado. Cambios en la infraestructura en la ciudad se harán necesarios (como más rampas, veredas más anchas, más parques, etc.), así como nuevos servicios públicos para esta población (más seguridad, más transporte público, mayor presencia de servicios de salud y de emergencia, etc.), sin contar con la estructuración de un sistema de pensiones para una mayor carga. No solo esta transformación será significativa en términos de cómo se verán las ciudades: será también significativa en términos de cuáles serán los grupos e intereses mayoritarios. Dichos cambios empiezan a ser notorios en algunos distritos en donde la población adulta mayor es significativa. En ellos, las municipalidades movilizan una parte de sus recursos a atender a población mayor, implementando distintos talleres (tejido, computación, artes, memoria, etc.) y servicios (belleza, salud, actividades deportivas y recreativas, entre otros). En el futuro, la población adulta mayor no solo crecerá en número, sino que tendrá tiempo y, con ello, capacidad de organizarse.

\section{REFERENCIAS BIBLIOGRÁFICAS}

Arellano, Rolando y David Burgos (2010). Ciudad de los Reyes, de los Chávez, de los Quispe. Lima: Planeta.

Borja, Jordi (2003). La ciudad conquistada. Buenos Aires: Alianza.

Bourdieu, Pierre (1984) [1979]. Distinction: A Social Critique of the Judgement of Taste. Cambridge MA: Harvard University Press.

Burawoy, Michael (1998). The Extended Case Method. Sociological Theory, 16(1), 4-33. https://doi.org/10.1111/0735-2751.00040

Centner, Ryan (2010). Spatializing Distinction in Cities of the Global South: Volatile Terrains of Morality and Citizenship. Political Power and Social Theory, 21, 281-298. https://doi.org/10.1108/S0198-8719(2010)0000021016

Centner, Ryan (2013). Distinguishing the Right Kind of City: Contentious Urban Middle Classes in Argentina, Brazil and Turkey. En Tony Samara, He Shenjing y Gou Chen (eds.), Locating Right to the City in the Global South (pp. 247-263). Londres, Nueva York: Routledge.

Comaroff, John (1982). Dialectical Systems, History and Anthropology: Units of Study and Questions of Theory. Journal of Southern African Studies, 8(2), 143-172. https://doi.org/10.1080/03057078208708040 
Cotlear, Daniel (ed.) (2011). Population Aging: Is Latin America Ready? Washington DC: The World Bank.

Da Silva, Graziela y Elisa Reis (2011). Perceptions of Racial Discrimination among Black Professionals in Rio de Janeiro. Latin American Research Review, 46(2), 55-78. https://doi.org/10.1353/lar.2011.0026

Elias, Norbert y John L. Scotson (1994) [1964]. The Established and the Outsiders: An Inquiry into Community Problems. Londres: Sage.

Franco, Rolando, Arturo León y Raúl Atria (eds.) (2007). Estratificación y movilidad social en América Latina: Transformaciones estructurales de un cuarto de siglo. Santiago de Chile: LOM, CEPAL, GTZ.

Franco, Rolando, Martin Hopenhayn y Arturo León (eds.) (2010). Las clases medias en América Latina: retrospectiva y nuevas tendencias. México DF: CEPAL, Siglo XXI.

Graves, Erin (2010). The Structuring of Urban Life in a Mixed-income Housing Community. City \& Community, 9(1), 109-131. https://doi.org/10.1111/j.15406040.2009.01305.x

Harvey, David (2006). Political Economy of Public Space. En Setha Low y Neil Smith (eds.), The Politics of Public Space (pp. 35-48). Nueva York, Londres: Routledge.

Hirschman, Albert (1970). Exit, Voice, and Loyalty: responses to decline in firms, organizations, and states. Cambridge, MA: Harvard University Press.

Joseph, Mark (2008). Early Resident Experiences at a New Mixed-Income Development in Chicago. Journal of Urban Affairs, 30(2), 229-257. https://doi.org/10.1111/ j.1467-9906.2008.00394.x

Lefebvre, Henri (1996 [1967]). The Right to the City. En Henri Lefebvre, Writings on Cities. Malden, Oxford, Carlton: Blackwell.

Logan, John y Harvey Molotch (1987). Urban Fortunes. The Political Economy of Place. Berkeley, Los Ángeles, Londres: University of California Press.

Méndez, María Luisa (2008). Middle-Class Identities in a Neoliberal Age: Tensions between Contested Authenticities. The Sociological Review, 5(2), 220-237. https://doi.org/10.1111/j.1467-954X.2008.00785.x

Pereyra, Omar (2015). Contemporary Middle Class in Latin America: A Study of San Felipe. Lanham: Lexington Books.

Portes, Alejandro y John Walton (1976). Urban Latin America: The Political Condition from Above and Below. Austin, Londres: The University of Texas Press. 
Portocarrero, Gonzalo (1998). Introducción. Ajuste de cuentas: las clases medias en el trabajo de Tempo. En Gonzalo Portocarrero (ed.), Las clases medias: entre la pretensión y la incertidumbre (pp. 9-37). Lima: Oxfam, Sur.

Roberts, Bryan (1978). Cities of Peasants: The Political Economy of Urbanization in the Third World. Beverly Hills, Londres: Sage Publications.

Saad, Paulo (2011). Demographic Trends in Latin America and the Caribbean. En Daniel Cotlear (ed.), Population Aging: Is Latin America Ready? (pp. 43-77). Washington DC: The World Bank.

Small, Mario Luis (2004). Villa Victoria: The Transformation of Social Capital in a Boston Barrio. Chicago: The University of Chicago Press. https://doi.org/10.7208/ chicago/9780226762937.001.0001

Snow, David y Robert Benford (1992). Master Frames and Cycles of Protest. En Morris, Aldon y Carol McClurg Mueller (eds.), Frontiers in Social Movement Theory (pp. 133-155). New Haven: Yale University Press. 